\title{
Analysis of the Contribution of the Development of Learning and Effectiveness Paradigm" to Multinational Corporations
}

\author{
Linyue Zhang
}

Beijing RDFZ Chaoyang branch school

Zhanglinyue@rdfzcygj.cn

\begin{abstract}
This paper aims to fully epitomize the development of the learning-and-effectiveness paradigm, from two other diversity management ways: the discrimination-and-fairness paradigm and the access-and-legitimacy paradigm. The Learning-and-effectiveness paradigm embraces the assimilation and differentiation between the workforce at the same time, which undoubtedly contribute to modern diversity management. Then, the benefits these changes may bring to the multinational companies, which are the main groups of businesses that adopt diversity management. Throughout this whole transforming process, the limitations derived from the former two approaches account for the shifts to a certain extent. Thus, the paper attempts to elaborate on the weaknesses of the first two paradigms first before introducing the benefits of the learning-and-effectiveness paradigm on its own. Additionally, the precautions or steps for using this paradigm are also mentioned in the paper, which should be considered by MNCs. At last, this paper summarized the main benefits the learning-and-effectiveness can bring to MNCs with any potentials for further research.
\end{abstract}

Keywords: Multinational companies, Learning-and-effectiveness paradigm, Discrimination-and-fairness paradigm, Access-and-legitimacy paradigm

\section{INTRODUCTION}

Human resource management is undoubtedly a crucial department in an organization, which aims to ensure the appropriate workforce are recruited, thereby contribute their skills to promote the further growth of the business. By definition, diversity management should be included as HRM's one task based on its wide range of policies relevant to recruitment and motivation. At the same time, the adoption of any techniques of diversity management can benefit businesses in different way. This paper, based on the above background information, tends to analyze one specific paradigm of diversity management, which is called the learning-and-effectiveness paradigm.

This paper will provide answers to several research questions. First of all, how can learning-and-effectiveness paradigm stand out compare with basic well-known diversity management channels? Those channels are referred to the former two approaches: the discrimination-and-fairness paradigm, as well as the access-and-legitimacy paradigm. In order to answer this question, it is necessary to first differentiate the learning-and-effectiveness paradigm from these two, followed by the detailed explanations account for the problems of them. Another basic question is that, are there any precautions when using this type of diversity management? When taking this, certain preparations need to be made in advance to ensure high efficiency in achieving expected targets. Therefore, the necessary steps before using learning-and-effectiveness paradigm will be listed in this paper with elaborations and examples given.

As a result, the paramount part of this paper is to epitomize the benefits of learning-and-effectiveness paradigm based on the above analysis on the importance of transformation. After answering all of these research questions, this paper can act as a meaningful tool in today's multinational corporations' management field. 


\section{OVERVIEW OF SEVERAL DIVERSITY MANAGEMENT}

Diversity management is widely believed as one of the crucial strategies for arousing and maintaining the morale of workers from different backgrounds. Discrimination, on the opposite side, leads to demotivation, absenteeism, social unrest, even health problems to a greater extent. One longitudinal research conducted in 2016 examined the influences of interpersonal discrimination on workers doing research in the STEM field [1]. The independent variable (IV) in this research included the extent of promotion in the academic rank and the social isolation. The dependent variables (DV) were measured through an objective measure of productivity based on the citation amounts, and the organizational citizenship behavior (OCB). As a result, the workers' voluntary commitment to work decreased, correlated with lower job satisfaction. In order to avoid this from happening in the early era, the paradigms of diversity management, including the discrimination-and-fairness paradigm, the access-and-legitimacy-paradigm, and the learning-and-effectiveness paradigm emerged in the 20 th century [2]. Each of them will be elaborated as follows.

The Discrimination-and-fairness paradigm and access-and-legitimacy paradigm can be considered as predecessors of the learning-and-effectiveness paradigm. In simple terms, the former embraces the idea of assimilation, while the latter believes the differentiation is a matter [3]. The discrimination-and-fairness paradigm that came up in the 1960s was clearly affected by the law. For example, the fairly housing act published in 1968 restricts the discrimination relevant to resales and rental housing [4]; the civil rights act of 1964 states that discrimination in firing and hiring is prohibited strictly [5]. Thus, the invention of the discrimination-and-fairness paradigm was mostly to prevent the businesses at that time from acting against law. To fulfill the assimilation, the diverse employees in the workplace should behave and get treated in the same way, which includes fair hiring or firing, and the same amounts of workloads assigned.

The access-and-legitimacy paradigm invented in the 1980s then began to view the diverse employees as resources for boosting business activities. Comparing with the discrimination-and-fairness paradigm, this tends to differentiate the workers and match them with a variety of clients accordingly. For example, cultural differences can act as an enormous problem for negotiating based on the challenges of communication between workers from a different country. For one thing, through direct or indirect communication, or high context and low context culture that will be explained deeper in the following paper. The negotiators in western countries merely embedded the context in their words, but explicitly express their preferences [6]. For another thing, the different habits in decision making may hinder their productivity. Under this situation, the access-and-legitimacy paradigm can distribute workers with distinct backgrounds to different segments of clients and negotiators, which slower the decision-making process thereby gain competitive advantages.

The development of the learning and effectiveness paradigm maximized the integration between assimilation and differentiation, which embody the belief that all employees should be incorporated in achieving the organization's objective even though they were assigned certain tasks toward their own 'segments'. For instance, if a France worker is hired for negotiating with the main France clients this multinational business is targeting, she will also feel free to come up with ideas in other fields in business, like the design for new advertisements. The diverse workers hired reflect the celebration of different, while the same chances in engaging in business decision making embody the assimilation between them. With this development process described, it is important to note that the changes and invention of new paradigms were not considered as groundless, since the discrimination-and-fairness paradigm and access-and-legitimacy paradigm both reveal their own defects when taken by MNCs.

\section{PROBLEMS WITH FORMOR APPROACHES-----LEAD TO THE FORMATION OF LEARNING-AND-EFFECTIVENESS
PARADIGM}

\subsection{The problems of discrimination-and-fairness paradigm}

One of the limitations of the discrimination-and-fairness paradigm is derived from the inability of firms to spot the chances to generate higher market share and profit. To be more specific, the external business activities may not benefit from the practice of using the discrimination-and-fairness paradigm. Because all employees are treated in the same way, the diversified workers were merely fully utilized by targeting them to different groups of clients and tasks. The motto for this paradigm is 'we are all the same, difference do not matter' [7]. However, at the same time, businesses should take advantage of diversified workers in diverse business cases, or this practice will hinder productivity. According to McKinsey's study of diversity, which found out that the companies in the top quartile of ethnic diversity generated $33 \%$ higher profit than companies in the low quartile [8]. Regarding this case, the employees from different backgrounds are more likely to negotiate 
peacefully with the clients from the same cultural background with them, so that fewer conflicts will ensure the working efficiency as a result. Thus, the discrimination-and-fairness paradigm enables all employees to perform in the same way, doing identical tasks, which certainly loses the chances of profit and efficiency maximization.

Additionally, even though the discrimination-and-fairness paradigm promotes assimilation, tensions are still existing in the internal workers' group. The conflicts arise from everywhere, including the distinct working habits derived from different cultural backgrounds, the explicit or implicit communication as well as troubles of different accents and fluency when there is a conversation. The high context and low context cultures can serve as good examples. High context culture's communications are indirect and layered, with tons of verbal and nonverbal cues. Whereas low-context communication stands on the opposite, with explicit and precise expressions of ideas and decisions [9]. The workers with low context habits might regard high context conversation as ineffective, lack of transparency and undisciplined. This is only one type of inner tension between workers, the larger disputes relevant to decision making can even become more detrimental to one organization. The cultures impact decision making to a larger extent. Japanese decision making is based on the harmony of the group, which is in common with the Chinese's process where individuals are usually maintained on their own position, avoid challenging authority and rushing the decision-making process [10]. But American usually feel free to questioning the authority and keep moving things along, which embody the difference that leads to the inner tensions inside the cultural assimilation.

\section{2 the problems of access-and-legitimacy paradigm}

Moving to the weaknesses of the access-and-legitimacy paradigm, which alleviates some problems triggered by the discrimination-and-fairness paradigm but brings out different issues. One problem when using this paradigm is that diverse workers are only responsible for their own works instead of having a holistic view of the organization. The France workers are only in charge of negotiating with groups of France clients, but neglecting the advertisements posted in other countries by other culture teams. This means that no one can replace their works if there were some emergency changes, which becomes a huge problem in a business's operation process. After the works done by the cultural teams, businesses embrace the success without learning from the cases, like the attitudes of clients in promotion and distribution channels or the norms for decision-making. As a result, new business activities and collaborations cannot get pursued without the cultural teams' existence, which triggers inefficiency and low profit generated in long run.

Moreover, the problems relating to demotivation can be regarded as a second large problem. For one thing, the use of the access-and-legitimacy paradigm may put more emphasis on cultural teams that spot more opportunities and grasp more market share for the business. After the achievements made, it is reasonable for the business to invest more in relevant departments. The business dominated by white teams employ some cultural teams, which continuously brought profits and opportunities for expansion to business. What business could do is reinvest in these departments to encourage further growth. At the same time, the white teams must be frustrated by the decision and became disincentivized to a greater extent. For another circumstance, the cultural team might become demotivated as well if they carried most of the business's workload. The research done in 2019 relevant to workload and motivation reflected that the correlation between these two is positive [11]. When a business finds out the possible achievement they could reach thereby exploiting and burdening them by setting out large amounts of tasks, the goal of improving productivity and incentives is backfired.

The development of the learning-and-effectiveness paradigm thereby minimizes the risks triggered by the above weaknesses of the discrimination-and-fairness paradigm as well as the access-and-legitimacy paradigm. By shifting the former two paradigms into this new one, all diverse workforces will work toward the same business objectives albeit they have their own works in assigned 'niche market'. That reduces the number of marginalized workers and boost business activities at the same time.

\section{NECESSARY STEPS MNCS SHOULD TAKE WHEN UTILIZING THE LEARNING-AND-EFFECTIVENESS PARADIGM}

To start with, it is important for a business to set out one mission and periodic objectives relevant to it for assimilating the cultural teams. According to the above explanations, a clear and precise goal can guide the diverse workers working toward it by providing their own suggestions and practices. Not only for the achievement of assimilation but objectives can also be utilized as a useful motivator in boosting the morale of workers. Psychology experts in Florida state university demonstrated that the goal settings are largely correlated with the individual's behavior [12]. The passages epitomized that the objectives should be set in the 'SMART' way, which is specific, measurable, attainable, relevant and time bounded. By their English meanings, one time-specific objective should be realistic 
that is achievable by the employees, which the achievements can also be measured in figures. The achievements made from this attainable goal motivates the diverse workers to pursue their further works with the joys derived from self-esteemed and self-actualization. These two keywords are the top two motivation levels based on Maslow's hierarchy of needs [13], which conveys the motivations obtained through the recognition of employees' status and the utilizing of skills. The continuous contributions made by the diverse employees in the business due to the setting of objectives fulfill the basic step of fulfilling the assimilation in the learning-and-effectiveness paradigm.

The adoption of one same language must be necessary for both assimilation and differentiation in a multinational company, which is typically English as a worldwide language. From the research paper global business speaks English by Tsedal Neeley, one in every four people worldwide grasp the abilities to speaking English at a useful level [14]. The author proposed that 5000 English vocabularies are fairly enough to ensure the effectiveness and efficiency in communication between workers in MNCs. To implement this, all of the non-native speakers must comply with the practice of English adoption in business, which is a matter since it takes time to convert their own language to English in a conversation. The adoption of English can ensure the learning of different habits from diverse clients through the discussions lead by the diverse employees that have taken part in the negotiations. The same language used reduces the risks of triggering asymmetrical information, thereby improving the efficiency inside the organization. The asymmetrical information can be considered as the problem of the access-and-legitimacy paradigm, where cultural teams have no idea of what each other teams are executing but only focusing on their own tasks. In order to implement the learning-and-effectiveness paradigm, this problem should be alleviated for sure.

Lastly, the tensions and conflicts between employees should be minimized when implementing the learning-and-effectiveness paradigm, which is because assimilation through the exchange of ideas can promote the achievement of common objectives. For example, the experience of one cultural team in dealing with groups of unfamiliar foreign clients. With the setting of mission and objectives, employees that have adopted the same language will feel free to discuss their own cases with each other under a peaceful atmosphere. On the opposite, if the organization has a bureaucratic structure between cultural teams, it is less likely for employees to share their own works and experiences flexibly with others in a competitive environment. Therefore, MNCs should try to develop a nonbureaucratic and uncompetitive environment for workers in order to implement the learning-and-effectiveness paradigm.
Multinational companies can benefit from using this paradigm in several ways, not limited to the basic gain in profits and productivity. The last part of this research paper tends to encapsulate the main welfares MNCs can reap from this practice.

\section{MAIN BENEFITS OF USING LEARNING-AND-EFFECTIVENESS PARADIGM}

\section{1 attain wider information}

One of the advantages is relevant to the wider information gain through diversified employees. The information stated here is not simply referred to the financial conditions and other current conditions of clients or business, but also include the taste, habits, as well as the characteristics of the groups. These pieces of information can be used in researching and developing new products and services delivered by this company. Coca-Cola Inc. can serve as a suitable example here, where the research done on consumers' tastes has driven up the revenue earned. The invention of 'Coke Zero' after noticing customers worldwide was more likely to purchase the healthier drinks pushed the growth of North American organic revenue by 3\% [15]. The clients in a different country have different habits and need when consuming for one product. By identifying the tastes and needs of diverse clients, the businesses have more chances to reinvest appropriately in the products to meet their wants.

\section{2 diversified workforce}

Moreover, using the learning-and-effectiveness paradigm enable multinational companies to have better access to diversified skilled workers in relevant field. The egalitarian environment created through this paradigm lets new workers feel welcome to a larger extent. The job seekers are less likely to apply for businesses that face tons of lawsuits of discrimination and gain a bad reputation due to that [16]. If this is happening, less high skilled labor will seek a place and contribute to the low reputable firm, especially when they are from a diverse background that might be the victims of discrimination. High-skilled laborers are usually highly educated in the relevant fields, who can contribute amounts of helpful ideas that can positively affect the firm. When the learning-and-effectiveness paradigm is taken, the nonbureaucratic atmosphere will be appealing for a variety of high skilled workers, getting recruited thereby working toward the objectives effectively.

\section{3 workers motivation}

Motivation can also be an obvious benefit there, as workers are one of the most crucial stakeholders in a 
business. Not limited to the incentives generated from working toward an objective, security needs, as well as social needs, are two relevant motivators under the case of using the learning-and-effectiveness paradigm. Security need, according to Maslow's hierarchy of need, can be referred to the safety at work from the workers' perspective. Even though the discrimination-and-fairness paradigm promotes assimilation, the color-blind environment provides an implicit idea of 'we are the same'. Thus, inner discrimination might still exist if some potential workers are racist. The access-and-legitimacy paradigm might not fulfill the job security, which can be considered as the stable income obtained and the possibility of getting redundant. If one cultural team resigned from the business, it takes time and costs for a new team to get access to the unfamiliar information. As a result, the higher costs might lead to worker redundancy or automation for reducing the direct costs. Social need or belonging need is at the third level of Maslow's hierarchy of need. The learning-and-effectiveness paradigm embodies the collaboration between cultural teams and outside teams. The interaction between workers within a peaceful, nonbureaucratic environment can motivate the workers for sure due to the obtaining of belonging need. Undoubtfully, the incentivized workers bring tons of benefits to a multinational business.

The benefits gained from the development of this paradigm can provide chances to MNCs for further expansion and recruitment of experts according to the above analysis.

\section{CONCLUSION}

To wrap up, this paper first provided a basic definition for the learning-and-effectiveness paradigm and the two former forms of paradigm. The difference between them wasclearly described and explained. Then, the thesis moved forward to the main part where the reasons for transformation in diversity management paradigms were elaborated. This transformation compensates for the low profitability triggered by the discrimination-and-fairness paradigm and demotivation caused by the access-and-legitimacy paradigm. In order to execute the learning-and-effectiveness paradigm, it is important for business to set the mission and objectives, adopt the same language, also eliminate the bureaucratic structure. This diversity management style can benefit multinational corporations through wider information gain, higher quality workers recruited with better motivation levels. But the problem arises, is this paradigm the best choice for all of the MNCs in the world? In order to find out the answer, further research is on the way.

This paper puts more emphasis on analyzing the learning-and-effectiveness paradigm from the relevant research done on this. My further research will be conducted in the form of a case study, which will be focusing on a single multinational firm. Did this firm partly or fully used this paradigm in managing diversity, how? If the firm is using the discrimination-and-fairness paradigm or access-and-legitimacy paradigm, a comparison can be made under this case between the paradigms of diversity management. Further research will not purely analyze the benefits of the learning-and-effectiveness paradigm, but also considering the facts that whether this paradigm is suitable for all types of multinational companies. Even though the existing information included in this paper is insufficient to provide a definite answer about the suitability of this paradigm for MNCs, the analysis made in this essay seems to provide a grand prospect for future research.

\section{ACKNOWLEDGMENT}

Firstly, I would like to show my deepest gratitude to my teachers and professors in my high school, who have provided me with guidance in choosing the topic and further relevant resources finding for this paper. Most importantly, my advisor viewed my paper and gave me valuable suggestions in improving the thesis.

I also would like to thank all my friends and parents for their encouragement and support, in particular my friend in university who recommended me to read some relevant articles. Without all their enlightening instruction and impressive kindness, I could not have completed my thesis.

\section{REFERENCES}

[1] O’Brien, K. R., McAbee, S. T., Hebl, M. R., \& Rodgers, J. R. (2016), The Impact of Interpersonal Discrimination and Stress on Health and Performance for Early Career STEM Academicians. Frontiers. https://www.frontiersin.org/articles/10.3389/fpsyg. 2016.00615/full\#B42

[2] Kwon, \& Nicolaides. (2017), Managing Diversity Through Triple-Loop Learning: A Call for Paradigm Shift. Human Resource Development Review, 85-88.

[3] David A. T, Robin J. Ely (2016), Making Differences Matter: A New Paradigm for Managing Diversity. Harvard Business Review, 52-60.

[4] History.com Editors. (2021). Fair Housing Act. Retrieved from https://www.history.com/topics/black-history/fair-h ousing-act

[5] Legal Highlight: The Civil Rights Act of 1964. United States Department of Labor. Retrieved from 
https://www.dol.gov/agencies/oasam/civil-rights-ce nter/statutes/civil-rights-act-of-1964

[6] Jeanne B et al. (2020) Managing Multicultural Teams. Harvard Business Review. 18-21

[7] Karencwise. (2011). The Three Paradigms of Equality \& Diversity. Karen Wise. Retrieved from https://karenwise.wordpress.com/2011/02/04/the-th ree-paradigms-of-equality-diversity/

[8] McKinsey\&company. (2018), deliverin -through diversity full report. https://www.mckinsey.com/ /media/mckinsey/busi ness \%20functions/organization/our\%20insights/del ivering\%20through\%20diversity/delivering-throug h-diversity_full-report.ashx

[9] Bansal, V. (2021). High Context Culture vs Low Context Culture: Communication Design for Avoiding Uncertainty. TechTello. Retrieved from https://www.techtello.com/high-context-culture-vslow-context-culture/

[10] Country Navigator. (2021). How do different cultures approach decision making? Country Navigator.

https://www.countrynavigator.com/blog/how-do-di fferent-cultures-approach-decision-making/

[11] I, A., \& D, R. (2019). Workload and Motivation on Employees Performance Analyzed by Information
Technology. $\quad$ Retrieved from https://www.researchgate.net/publication/33740128 8_Workload_and_Motivation_on_Employees_Perf ormance_Analyzed_by_Information_Technology

[12] Wroblewski, M. T. (2020). Importance of Motivation and Goal Setting for Businesses. Small Business - Chron.com. Retrieved from https://smallbusiness.chron.com/importance-motiva tion-goal-setting-businesses-2506.html

[13] Corporate Finance Institute. (2020) Maslow's Hierarchy of Needs - Overview, Explanation, and Examples. Retrieved from https://corporatefinanceinstitute.com/resources/kno wledge/other/maslows-hierarchy-of-needs/

[14]Tsedal, N. (2012) Global Business Speaks English. Harvard Business Review. 103-105

[15] Lucas, A. (2019). Coca-Cola says strong sales of Coke Zero Sugar are driving revenue growth. CNBC. Retrieved from https://www.cnbc.com/2019/10/18/coca-cola-ko-ea rnings-q3-2019.html

[16] Benjamin, T. (2016). The Ways Discrimination Negatively Affects Businesses. Small Business Chron.com. Retrieved from https://smallbusiness.chron.com/ways-discriminati on-negatively-affects-businesses-36925.html 